\title{
SOME PÓLYA-TYPE IRREDUCIBILITY CRITERIA FOR MULTIVARIATE POLYNOMIALS
}

\author{
NICOLAE CIPRIAN BONCIOCAT, YANN BUGEAUD, MIHAI CIPU, AND MAURICE MIGNOTTE
}

\begin{abstract}
We provide irreducibility criteria for multivariate polynomials with coefficients in an arbitrary field, that extend a classical result of Pólya for polynomials with integer coefficients. In particular, we provide irreducibility conditions for polynomials of the form $f(X)\left(Y-f_{1}(X)\right) \cdots\left(Y-f_{n}(X)\right)+g(X)$, with $f, f_{1}, \ldots, f_{n}, g$ univariate polynomials over an arbitrary field.
\end{abstract}

\section{INTRODUCTION}

There are many irreducibility criteria for multivariate polynomials in the literature, obtained by using various methods. Some of the classical techniques used in this respect require the study of Riemann surfaces and Puiseux expansions [17], the study of convex polyhedra [22], or the study of nonarchimedean absolute values over arbitrary fields [14] and [15].

Some more recent irreducibility results have been obtained for various classes of multivariate polynomials, such as difference polynomials [19], [16], [2] and [3], linear combinations of relatively prime polynomials [9], compositions of polynomials [1] and [6], multiplicative convolutions [5], polynomials having one coefficient of dominant degree [7], lacunary polynomials [4], and polynomials obtained from irreducible polynomials in fewer variables [8]. For an excellent account on the techniques used in the study of reducibility of polynomials over arbitrary fields, the reader is referred to Schinzel's book [20].

In [18] Pólya proved the following elegant irreducibility criterion for polynomials with integer coefficients.

Theorem (Pólya, 1919) If for $n$ integral values of $x$, the integral polynomial $f(x)$ of degree $n$ has values which are different from zero, and in absolute value less than

$$
\frac{\lceil n / 2\rceil !}{2^{\lceil n / 2\rceil},}
$$

then $f(x)$ is irreducible over $\mathbb{Q}$.

The proof of this irreducibility criterion relies on the following lemma.

Lemma (Pólya, 1919) Let $f(x)$ be an integral polynomial of degree $n$. Then among any pairwise distinct integers $x_{0}, \ldots, x_{n}$ there exists at least one, say $x_{i}$, such that $\left|f\left(x_{i}\right)\right| \geq n ! / 2^{n}$.

2010 Mathematics Subject Classification. Primary 11R09; Secondary 11C08.

Key words and phrases. irreducible multivariate polynomials, nonarchimedean absolute value. 
The aim of this paper is to provide several irreducibility criteria for multivariate polynomials, that extend in a natural way Pólya's Theorem. The first result we will prove is the following irreducibility criterion for bivariate polynomials over an arbitrary field.

Theorem 1.1. Let $K$ be a field and $F(X, Y) \in K[X, Y]$ with $\operatorname{deg}_{Y} F(X, Y)=n$. If for $n$ polynomials $c_{1}, \ldots, c_{n} \in K[X]$ with pairwise distinct degrees one has

$$
0 \leq \operatorname{deg} F\left(X, c_{i}(X)\right)<\frac{\lceil n / 2\rceil(\lceil n / 2\rceil-1)}{2}, \quad i=1, \ldots, n,
$$

then $F(X, Y)$ is irreducible over $K(X)$. The same conclusion holds if

$$
0 \leq \operatorname{deg} F\left(X, c_{i}(X)\right)<\frac{\lceil n / 2\rceil(\lceil n / 2\rceil+1)}{2}, \quad i=1, \ldots, n,
$$

provided $c_{1}, \ldots, c_{n} \in K[X]$ are non-zero polynomials with pairwise distinct degrees.

Let us note here that (1) makes sense only for $n \geq 3$, while (2) makes sense for $n \geq 2$. By Theorem 1.1, one may immediately deduce the following result.

Corollary 1.2. Let $K$ be a field. For any polynomials $f_{1}, \ldots, f_{n} \in K[X]$ with pairwise distinct degrees, and any non-zero polynomials $f, g \in K[X]$ with

$$
\operatorname{deg} g<\frac{\lceil n / 2\rceil(\lceil n / 2\rceil-1)}{2},
$$

the polynomial $F(X, Y)=f(X)\left(Y-f_{1}(X)\right) \cdots\left(Y-f_{n}(X)\right)+g(X)$ is irreducible over $K(X)$. The same conclusion holds if

$$
\operatorname{deg} g<\frac{\lceil n / 2\rceil(\lceil n / 2\rceil+1)}{2}
$$

provided $f_{1}, \ldots, f_{n} \in K[X]$ are non-zero polynomials with pairwise distinct degrees.

We note here that there are many classical irreducibility criteria for polynomials of the form $a_{0}\left(X-a_{1}\right) \cdots\left(X-a_{n}\right)+a_{n+1}$, where the $a_{i}$ are rational integers. Many of these criteria depend upon the arithmetical properties of the values taken by such a polynomial at some integral arguments. The first criterion of this kind was suggested by Schur [21], who raised the question of the irreducibility of the polynomials of the form $\left(X-a_{1}\right) \cdots\left(X-a_{n}\right) \pm 1$. For a unifying approach of the irreducibility criteria for polynomials of this type, we refer the interested reader to [10], [11] and [12].

Another immediate consequence of Theorem 1.1 is a similar result for polynomials in $r \geq 3$ variables $X_{1}, X_{2}, \ldots, X_{r}$ over $K$. For any polynomial $f \in K\left[X_{1}, \ldots, X_{r}\right]$ we denote by $\operatorname{deg}_{r} f$ the degree of $f$ as a polynomial in $X_{r}$ with coefficients in $K\left[X_{1}, \ldots, X_{r-1}\right]$. The next result follows from Theorem 1.1 by writing $Y$ for $X_{r}, X$ for $X_{r-1}$ and by replacing $K$ with $K\left(X_{1}, \ldots, X_{r-2}\right)$. 
Theorem 1.3. Let $K$ be a field, $r \geq 3$, and let $F\left(X_{1}, \ldots, X_{r}\right) \in K\left[X_{1}, \ldots, X_{r}\right]$ with $\operatorname{deg}_{r} F=n$. If for $n$ polynomials $c_{1}, \ldots, c_{n} \in K\left[X_{1}, \ldots, X_{r-1}\right]$ with $\operatorname{deg}_{r-1} c_{1}, \ldots, \operatorname{deg}_{r-1} c_{n}$ pairwise distinct, one has

$$
0 \leq \operatorname{deg}_{r-1} F\left(X_{1}, \ldots, X_{r-1}, c_{i}\right)<\frac{\lceil n / 2\rceil(\lceil n / 2\rceil-1)}{2}, \quad i=1, \ldots, n,
$$

then $F$ is irreducible over $K\left(X_{1}, \ldots, X_{r-1}\right)$. The same conclusion holds if

$$
0 \leq \operatorname{deg}_{r-1} F\left(X_{1}, \ldots, X_{r-1}, c_{i}\right)<\frac{\lceil n / 2\rceil(\lceil n / 2\rceil+1)}{2}, \quad i=1, \ldots, n,
$$

provided $c_{1}, \ldots, c_{n}$ are non-zero polynomials with $\operatorname{deg}_{r-1} c_{1}, \ldots, \operatorname{deg}_{r-1} c_{n}$ pairwise distinct.

For the proof of Theorem 1.1 we will need the following analogue of Pólya's Lemma.

Lemma 1.4. Let $K$ be a field and $F(X, Y) \in K[X, Y]$ with $\operatorname{deg}_{Y} F(X, Y)=n$. Then among any $n+1$ polynomials $c_{0}, \ldots, c_{n} \in K[X]$ with pairwise distinct degrees there exists at least one, say $c_{i}$, such that $\operatorname{deg} F\left(X, c_{i}(X)\right) \geq n(n-1) / 2$. Moreover, among any $n+1$ non-zero polynomials $c_{0}, \ldots, c_{n} \in K[X]$ with pairwise distinct degrees, there exists at least one, say $c_{i}$, such that $\operatorname{deg} F\left(X, c_{i}(X)\right) \geq n(n+1) / 2$.

In fact, a result similar to Lemma 1.4 holds with less restrictive conditions on the degrees of the polynomials $c_{0}, \ldots, c_{n}$, as follows.

Lemma 1.5. Let $K$ be a field and let $F(X, Y) \in K[X, Y]$ with $\operatorname{deg}_{Y} F(X, Y)=n$. Then among any $n+1$ polynomials $c_{0}, \ldots, c_{n} \in K[X]$ with pairwise distinct leading coefficients and $\operatorname{deg} c_{0} \leq \operatorname{deg} c_{1} \leq \cdots \leq \operatorname{deg} c_{n}$, there exists at least one, say $c_{i}$, such that $\operatorname{deg} F\left(X, c_{i}(X)\right) \geq$ $\operatorname{deg} c_{1}+\cdots+\operatorname{deg} c_{n}$.

An immediate application of Lemma 1.5 gives the following related irreducibility criterion.

Theorem 1.6. Let $K$ be a field and let $F(X, Y) \in K[X, Y]$ with $\operatorname{deg}_{Y} F(X, Y)=n$. If for $n$ polynomials $c_{1}, \ldots, c_{n} \in K[X]$ with $\operatorname{deg} c_{1} \leq \operatorname{deg} c_{2} \leq \cdots \leq \operatorname{deg} c_{n}$ and pairwise distinct leading coefficients one has

$$
0 \leq \operatorname{deg} F\left(X, c_{i}(X)\right)<\operatorname{deg} c_{2}+\operatorname{deg} c_{3}+\cdots+\operatorname{deg} c_{\lceil n / 2\rceil+1}, \quad i=1, \ldots, n,
$$

then $F$ is irreducible over $K(X)$.

Note that in some cases the price payed for using less restrictive conditions on the degrees of $c_{1}, \ldots, c_{n}$ may consist of stronger conditions on the degrees of $F\left(X, c_{1}(X)\right), \ldots, F\left(X, c_{n}(X)\right)$.

Corollary 1.7. Let $K$ be a field. For any polynomials $f_{1}, \ldots, f_{n} \in K[X]$ with pairwise distinct leading coefficients and $\operatorname{deg} f_{1} \leq \operatorname{deg} f_{2} \leq \cdots \leq \operatorname{deg} f_{n}$, and any non-zero polynomials $f, g \in K[X]$ with

$$
\operatorname{deg} g<\operatorname{deg} f_{2}+\operatorname{deg} f_{3}+\cdots+\operatorname{deg} f_{\lceil n / 2\rceil+1},
$$

the polynomial $F(X, Y)=f(X)\left(Y-f_{1}(X)\right) \cdots\left(Y-f_{n}(X)\right)+g(X)$ is irreducible over $K(X)$. 
By Theorem 1.6 we immediately obtain a similar result for polynomials in $r \geq 3$ variables $X_{1}, X_{2}, \ldots, X_{r}$ over $K$.

Theorem 1.8. Let $K$ be a field, $r \geq 3$, and let $F\left(X_{1}, \ldots, X_{r}\right) \in K\left[X_{1}, \ldots, X_{r}\right]$ with $\operatorname{deg}_{r} F=n$. If for $n$ polynomials $c_{1}, \ldots, c_{n} \in K\left[X_{1}, \ldots, X_{r-1}\right]$ with pairwise distinct leading coefficients with respect to $X_{r-1}$ and $\operatorname{deg}_{r-1} c_{1} \leq \operatorname{deg}_{r-1} c_{2} \leq \cdots \leq \operatorname{deg}_{r-1} c_{n}$ one has

$$
0 \leq \operatorname{deg}_{r-1} F\left(X_{1}, \ldots, X_{r-1}, c_{i}\right)<\operatorname{deg}_{r-1} c_{2}+\operatorname{deg}_{r-1} c_{3}+\cdots+\operatorname{deg}_{r-1} c_{\lceil n / 2\rceil+1}
$$

for each $i=1, \ldots, n$, then $F$ is irreducible over $K\left(X_{1}, \ldots, X_{r-1}\right)$.

The reader may naturally wonder how sharp the above results are. For instance, conditions (3) and (4) in Corollary 1.2 and condition (8) in Corollary 1.7 are best possible in the sense that there exist polynomials for which equality in (3) or (4), or (8) holds, and which are reducible over $K(X)$.

To see that the inequality sign in condition (3) can not be replaced by $\leq$ when the $c_{i}$ have pairwise distinct degrees, take for instance $K=\mathbb{Q}$ and $F_{1}(X, Y)=Y(Y-1)(Y-X)+2 X+2$. In this case we have $n=\operatorname{deg}_{Y} F_{1}=3, \frac{\lceil n / 2\rceil(\lceil n / 2\rceil-1)}{2}=1, f(X)=1, f_{1}(X)=0, f_{2}(X)=1$, $f_{3}(X)=X$ and $g(X)=2 X+2$. Since $\operatorname{deg} g=1$, we have equality in (3), while $F_{1}$ is obviously reducible, since $F_{1}(X, Y)=(Y+1) \cdot\left(Y^{2}-(X+2) Y+2 X+2\right)$.

Now, to see that the inequality sign in condition (4) can not be replaced by $\leq$ when the $c_{i}$ are non-zero polynomials with pairwise distinct degrees, let $K=\mathbb{Q}$ and consider the polynomial $F_{2}(X, Y)=(Y-1)(Y-X)-2 X-2$. Here $n=\operatorname{deg}_{Y} F_{2}=2, \frac{\lceil n / 2\rceil(\lceil n / 2\rceil+1)}{2}=1$, $f(X)=1, f_{1}(X)=1, f_{2}(X)=X$ and $g(X)=-2 X-2$. Since $\operatorname{deg} g=1$, we have equality in (4), while $F_{2}$ is reducible too, since $F_{2}(X, Y)=(Y+1) \cdot(Y-X-2)$.

For an example related to Corollary 1.7 , let $K=\mathbb{Q}$ and $F_{3}(X, Y)=(Y-X)(Y-2 X)-$ $2 X^{2}+3 X-1$. Here $n=2,\lceil n / 2\rceil+1=2, f(X)=1, f_{1}(X)=X, f_{2}(X)=2 X, \operatorname{deg} f_{1}=\operatorname{deg} f_{2}$ and $g(X)=-2 X^{2}+3 X-1$. We have equality in (8), while $F_{3}$ is obviously reducible, since $F_{3}(X, Y)=(Y-1) \cdot(Y-3 X+1)$.

Our results are quite flexible and may be used in various applications where other irreducibility criteria fail. The proofs of the main results are given in Section 2 below. We will also provide some examples of infinite families of irreducible polynomials in the last section of the paper.

\section{Proofs of the MAIN RESUlts}

2.1. Proof of Lemma 1.4. Let $c_{0}, \ldots, c_{n} \in K[X]$ be $n+1$ arbitrary polynomials with pairwise distinct degrees. By Lagrange's interpolation formula, we may write $F$ as

$$
F(X, Y)=\sum_{i=0}^{n} F\left(X, c_{i}\right) \cdot \frac{\left(Y-c_{0}\right) \cdots\left(Y-c_{i-1}\right)\left(Y-c_{i+1}\right) \cdots\left(Y-c_{n}\right)}{\left(c_{i}-c_{0}\right) \cdots\left(c_{i}-c_{i-1}\right)\left(c_{i}-c_{i+1}\right) \cdots\left(c_{i}-c_{n}\right)} .
$$

Note that (10) makes sense, since the $c_{i}$ are supposed to be pairwise distinct. By (10) we deduce that the leading coefficient of $F$, regarded as a polynomial in $Y$ with coefficients 
$a_{i} \in K[X]$, may be expressed as

$$
a_{n}(X)=\sum_{i=0}^{n} \frac{F\left(X, c_{i}\right)}{\left(c_{i}-c_{0}\right) \cdots\left(c_{i}-c_{i-1}\right)\left(c_{i}-c_{i+1}\right) \cdots\left(c_{i}-c_{n}\right)} .
$$

We now introduce a nonarchimedean absolute value $|\cdot|$ on $K(X)$, as follows. We fix an arbitrary real number $\rho>1$, and for any polynomial $u(X) \in K[X]$ we define $|u(X)|$ by the equality

$$
|u(X)|=\rho^{\operatorname{deg} u(X)} .
$$

We then extend the absolute value $|\cdot|$ to $K(X)$ by multiplicativity. Thus for any $w(X) \in$ $K(X), w(X)=\frac{u(X)}{v(X)}$, with $u(X), v(X) \in K[X], v(X) \neq 0$, we let $|w(X)|=\frac{|u(X)|}{|v(X)|}$.

Let us note that for any non-zero element $u(X)$ of $K[X]$ one has $|u(X)| \geq 1$. In particular, since $a_{n} \neq 0$, we have

$$
\left|a_{n}(X)\right| \geq 1
$$

The proof proceeds by reduction to absurd. Let us assume that $\operatorname{deg} F\left(X, c_{i}(X)\right)<n(n-1) / 2$ for each $i=0, \ldots, n$. Then, by (11) and the fact that our absolute value is nonarchimedean, we deduce that

$$
\begin{aligned}
\left|a_{n}(X)\right| & =\left|\sum_{i=0}^{n} \frac{F\left(X, c_{i}\right)}{\left(c_{i}-c_{0}\right) \cdots\left(c_{i}-c_{i-1}\right)\left(c_{i}-c_{i+1}\right) \cdots\left(c_{i}-c_{n}\right)}\right| \\
& \leq \max _{0 \leq i \leq n}\left|\frac{F\left(X, c_{i}\right)}{\left(c_{i}-c_{0}\right) \cdots\left(c_{i}-c_{i-1}\right)\left(c_{i}-c_{i+1}\right) \cdots\left(c_{i}-c_{n}\right)}\right| \\
& \leq \frac{\max _{0 \leq i \leq n}\left|F\left(X, c_{i}\right)\right|}{\min _{0 \leq i \leq n}\left|\left(c_{i}-c_{0}\right) \cdots\left(c_{i}-c_{i-1}\right)\left(c_{i}-c_{i+1}\right) \cdots\left(c_{i}-c_{n}\right)\right|} \\
& <\frac{\rho^{\frac{n(n-1)}{2}}}{\min _{0 \leq i \leq n}\left|\left(c_{i}-c_{0}\right) \cdots\left(c_{i}-c_{i-1}\right)\left(c_{i}-c_{i+1}\right) \cdots\left(c_{i}-c_{n}\right)\right|}
\end{aligned}
$$

Recall now that the degrees of $c_{0}, \ldots, c_{n}$ are pairwise distinct, so we may assume without loss of generality that $\operatorname{deg} c_{0}<\operatorname{deg} c_{1}<\cdots<\operatorname{deg} c_{n}$. In particular, this shows that $\operatorname{deg} c_{i} \geq i-1$ for $i=1, \ldots, n$. We then deduce that

$$
\begin{aligned}
\left|a_{n}(X)\right| & <\frac{\rho^{\frac{n(n-1)}{2}}}{\min _{1 \leq i \leq n}\left|c_{i}\right|^{i} \cdot\left|c_{i+1}\right| \cdots\left|c_{n}\right|}=\frac{\rho^{\frac{n(n-1)}{2}}}{\left|c_{1}\right| \cdot\left|c_{2}\right| \cdots\left|c_{n}\right|} \\
& =\frac{\rho^{\frac{n(n-1)}{2}}}{\rho^{\operatorname{deg} c_{1}+\operatorname{deg} c_{2}+\cdots+\operatorname{deg} c_{n}}} \leq \frac{\rho^{\frac{n(n-1)}{2}}}{\rho^{1+2+\cdots+(n-1)}}=1,
\end{aligned}
$$

which contradicts (12). This completes the proof of the first part of the lemma.

The proof of the second part of the lemma follows in a similar way, except that here the fact that $c_{0}, \ldots, c_{n} \in K[X]$ are non-zero polynomials with pairwise distinct degrees implies $\operatorname{deg} c_{i} \geq i$ for $i=0, \ldots, n$, which allows one to replace the bound $n(n-1) / 2$ by $n(n+1) / 2$. 
2.2. Proof of Lemma 1.5. Since $\operatorname{deg} c_{0} \leq \operatorname{deg} c_{1} \leq \cdots \leq \operatorname{deg} c_{n}$, our assumption that the $c_{i}$ have pairwise distinct leading coefficients shows that

$$
\operatorname{deg}\left(c_{i}-c_{j}\right)=\operatorname{deg} c_{\max \{i, j\}}
$$

for each pair of distinct indices $i, j$. On the other hand, the fact that the $c_{i}$ have pairwise distinct leading coefficients shows that the $c_{i}$ are pairwise distinct. In particular, at most one of the $c_{i}$, namely $c_{0}$, may be the zero polynomial.

Now, assuming that $\operatorname{deg} F\left(X, c_{i}(X)\right)<\operatorname{deg} c_{1}+\cdots+\operatorname{deg} c_{n}$ for each $i=0, \ldots, n$ and using the method employed in the proof of Lemma 1.4, we deduce that

$$
\left|a_{n}(X)\right|<\frac{\rho^{\operatorname{deg} c_{1}+\cdots+\operatorname{deg} c_{n}}}{\min _{0 \leq i \leq n}\left|\left(c_{i}-c_{0}\right) \cdots\left(c_{i}-c_{i-1}\right)\left(c_{i}-c_{i+1}\right) \cdots\left(c_{i}-c_{n}\right)\right|} .
$$

Using (13), we further obtain

$$
\left|a_{n}(X)\right|<\frac{\rho^{\operatorname{deg} c_{1}+\cdots+\operatorname{deg} c_{n}}}{\min _{1 \leq i \leq n}\left|c_{i}\right|^{i} \cdot\left|c_{i+1}\right| \cdots\left|c_{n}\right|}=\frac{\rho^{\operatorname{deg} c_{1}+\cdots+\operatorname{deg} c_{n}}}{\left|c_{1}\right| \cdot\left|c_{2}\right| \cdots\left|c_{n}\right|}=1,
$$

again a contradiction, and this completes the proof.

2.3. Proof of Theorem 1.1. First of all let us note that condition $0 \leq \operatorname{deg} F\left(X, c_{i}(X)\right)$ allows us to avoid the trivial case when $F$ is divisible by $Y-c_{i}(X)$. Now let us assume, for the sake of a contradiction, that $F$ as a polynomial in $Y$ with coefficients in $K[X]$ is reducible over $K(X)$. By the celebrated Lemma of Gauss we may further assume that $F=G \cdot H$, with $G, H \in K[X, Y], \operatorname{deg}_{Y} G \geq 1, \operatorname{deg}_{Y} H \geq 1$. Let now $m=\operatorname{deg}_{Y} G$. Without loss of generality we may assume that $\operatorname{deg}_{Y} G \geq \operatorname{deg}_{Y} H$, hence

$$
\lceil n / 2\rceil \leq m<n .
$$

Next, since $F\left(X, c_{i}(X)\right)=G\left(X, c_{i}(X)\right) H\left(X, c_{i}(X)\right)$ for each $i=1, \ldots, n$, we obtain

$$
\begin{aligned}
\operatorname{deg} G\left(X, c_{i}(X)\right) & \leq \operatorname{deg} F\left(X, c_{i}(X)\right) \\
& <\lceil n / 2\rceil(\lceil n / 2\rceil-1) / 2 \\
& \leq m(m-1) / 2, \quad i=1, \ldots, n .
\end{aligned}
$$

In particular, since $n \geq m+1$, we have

$$
\operatorname{deg} G\left(X, c_{i}(X)\right)<m(m-1) / 2, \quad i=1, \ldots, m+1 .
$$

On the other hand, according to Lemma 1.4, there must be an index $i \in\{1, \ldots, m+1\}$ such that $\operatorname{deg} G\left(X, c_{i}(X)\right) \geq m(m-1) / 2$, which contradicts (14), and this completes the proof of the first part of the theorem.

The second part of the proof follows in a similar way, except that instead of (14) one obtains

$$
\operatorname{deg} G\left(X, c_{i}(X)\right)<m(m+1) / 2, \quad i=1, \ldots, m+1,
$$

which cannot hold, according to Lemma 1.4. This completes the proof of the theorem. 
2.4. Proof of Theorem 1.6. Here too, the condition $0 \leq \operatorname{deg} F\left(X, c_{i}(X)\right)$ allows us to avoid the trivial case when $F$ is divisible by $Y-c_{i}(X)$. Let us assume as before that $F=G \cdot H$, with $G, H \in K[X, Y], m=\operatorname{deg}_{Y} G \geq \operatorname{deg}_{Y} H \geq 1$, so

$$
\lceil n / 2\rceil \leq m<n \text {. }
$$

Next, since $F\left(X, c_{i}(X)\right)=G\left(X, c_{i}(X)\right) H\left(X, c_{i}(X)\right)$ for each $i=1, \ldots, n$, we deduce that

$$
\begin{aligned}
\operatorname{deg} G\left(X, c_{i}(X)\right) & \leq \operatorname{deg} F\left(X, c_{i}(X)\right) \\
& <\operatorname{deg} c_{2}+\operatorname{deg} c_{3}+\cdots+\operatorname{deg} c_{\lceil n / 2\rceil+1} \\
& \leq \operatorname{deg} c_{2}+\operatorname{deg} c_{3}+\cdots+\operatorname{deg} c_{m+1}, \quad i=1, \ldots, n .
\end{aligned}
$$

In particular, since $n \geq m+1$, we have

$$
\operatorname{deg} G\left(X, c_{i}(X)\right)<\operatorname{deg} c_{2}+\operatorname{deg} c_{3}+\cdots+\operatorname{deg} c_{m+1}, \quad i=1, \ldots, m+1 .
$$

On the other hand, according to Lemma 1.5, there must be an index $i \in\{1, \ldots, m+1\}$ such that $\operatorname{deg} G\left(X, c_{i}(X)\right) \geq \operatorname{deg} c_{2}+\operatorname{deg} c_{3}+\cdots+\operatorname{deg} c_{m+1}$, which contradicts (15), and this completes the proof of the theorem.

We will also prove a $p$-adic version of Pólya's Lemma, which might be of independent interest. For a rational prime $p$ and an arbitrary nonzero rational number $r$, we will denote by $\nu_{p}(r)$ the exponent of $p$ in the prime decomposition of $r\left(\nu_{p}(0)=\infty\right)$. With this notation we have the following result.

Lemma 2.1. Let $F(X)=a_{0}+a_{1} X+\cdots+a_{n} X^{n} \in \mathbb{Z}[X]$, and let $p$ be a prime number. Then among any $n+1$ integers $c_{0}, c_{1}, \ldots, c_{n}$ with $\nu_{p}\left(c_{0}\right)>\nu_{p}\left(c_{1}\right)>\cdots>\nu_{p}\left(c_{n}\right)$ there exists at least one, say $c_{i}$, such that $\nu_{p}\left(F\left(c_{i}\right)\right) \leq \nu_{p}\left(a_{n}\right)+\nu_{p}\left(c_{1}\right)+\nu_{p}\left(c_{2}\right)+\cdots+\nu_{p}\left(c_{n}\right)$.

2.5 Proof of Lemma 2.1. We will first introduce a nonarchimedean absolute value $|\cdot|_{p}$ on $\mathbb{Q}$, as follows. For an arbitrary rational number $r$ we define $|r|_{p}=p^{-\nu_{p}(r)}$. This absolute value satisfies $|a b|_{p}=|a|_{p}|b|_{p}$ and $|a+b|_{p} \leq \max \left\{|a|_{p},|b|_{p}\right\}$, and it can be extended to a number field (see, for instance, [13]), but in our proof we will not need this feature of $|\cdot|_{p}$.

Let now $c_{0}, \ldots, c_{n} \in \mathbb{Z}$ be $n+1$ arbitrary integers with $\nu_{p}\left(c_{0}\right)>\nu_{p}\left(c_{1}\right)>\cdots>\nu_{p}\left(c_{n}\right)$, that is

$$
\left|c_{0}\right|_{p}<\left|c_{1}\right|_{p}<\cdots<\left|c_{n}\right|_{p}
$$

Using again Lagrange's interpolation formula, one may write $F$ as

$$
F(X)=\sum_{i=0}^{n} F\left(c_{i}\right) \cdot \frac{\left(X-c_{0}\right) \cdots\left(X-c_{i-1}\right)\left(X-c_{i+1}\right) \cdots\left(X-c_{n}\right)}{\left(c_{i}-c_{0}\right) \cdots\left(c_{i}-c_{i-1}\right)\left(c_{i}-c_{i+1}\right) \cdots\left(c_{i}-c_{n}\right)}
$$

Here too (17) makes sense, since the $c_{i}$ in the statement of Lemma 2.1 must be pairwise distinct, so the leading coefficient of $F$ may be expressed as

$$
a_{n}=\sum_{i=0}^{n} \frac{F\left(c_{i}\right)}{\left(c_{i}-c_{0}\right) \cdots\left(c_{i}-c_{i-1}\right)\left(c_{i}-c_{i+1}\right) \cdots\left(c_{i}-c_{n}\right)} .
$$


Now, let us assume that $\nu_{p}\left(F\left(c_{i}\right)\right)>\nu_{p}\left(a_{n}\right)+\nu_{p}\left(c_{1}\right)+\nu_{p}\left(c_{2}\right)+\cdots+\nu_{p}\left(c_{n}\right)$ for each $i=0,1, \ldots, n$, that is $\max _{0 \leq i \leq n}\left|F\left(c_{i}\right)\right|_{p}<\left|a_{n}\right|_{p} \cdot\left|c_{1}\right|_{p} \cdot\left|c_{2}\right|_{p} \cdots\left|c_{n}\right|_{p}$. Then, by (18) and the fact that our absolute value is nonarchimedean, we deduce that

$$
\begin{aligned}
\left|a_{n}\right|_{p} & =\left|\sum_{i=0}^{n} \frac{F\left(c_{i}\right)}{\left(c_{i}-c_{0}\right) \cdots\left(c_{i}-c_{i-1}\right)\left(c_{i}-c_{i+1}\right) \cdots\left(c_{i}-c_{n}\right)}\right|_{p} \\
& \leq \max _{0 \leq i \leq n}\left|\frac{F\left(c_{i}\right)}{\left(c_{i}-c_{0}\right) \cdots\left(c_{i}-c_{i-1}\right)\left(c_{i}-c_{i+1}\right) \cdots\left(c_{i}-c_{n}\right)}\right|_{p} \\
& \leq \frac{\max _{0 \leq i \leq n}\left|F\left(c_{i}\right)\right|_{p}}{\min _{0 \leq i \leq n}\left|\left(c_{i}-c_{0}\right) \cdots\left(c_{i}-c_{i-1}\right)\left(c_{i}-c_{i+1}\right) \cdots\left(c_{i}-c_{n}\right)\right|_{p}} \\
& <\frac{\left|a_{n}\right|_{p} \cdot\left|c_{1}\right|_{p} \cdot\left|c_{2}\right|_{p} \cdots\left|c_{n}\right|_{p}}{\min _{0 \leq i \leq n}\left|\left(c_{i}-c_{0}\right) \cdots\left(c_{i}-c_{i-1}\right)\left(c_{i}-c_{i+1}\right) \cdots\left(c_{i}-c_{n}\right)\right|_{p}} .
\end{aligned}
$$

Since for $i<j$ one has $\left|c_{i}-c_{j}\right|_{p}=\left|c_{j}\right|_{p}$, we then deduce that

$$
\left|a_{n}\right|_{p}<\frac{\left|a_{n}\right|_{p} \cdot\left|c_{1}\right|_{p} \cdot\left|c_{2}\right|_{p} \cdots\left|c_{n}\right|_{p}}{\min _{1 \leq i \leq n}\left|c_{i}\right|_{p}^{i} \cdot\left|c_{i+1}\right|_{p} \cdots\left|c_{n}\right|_{p}}=\frac{\left|a_{n}\right|_{p} \cdot\left|c_{1}\right|_{p} \cdot\left|c_{2}\right|_{p} \cdots\left|c_{n}\right|_{p}}{\left|c_{1}\right|_{p} \cdot\left|c_{2}\right|_{p} \cdots\left|c_{n}\right|_{p}}=\left|a_{n}\right|_{p},
$$

which is a contradiction. This completes the proof of the lemma.

We end this section by noting that no immediate application of Lemma 2.1 seems to be useful to derive similar irreducibility conditions in a $p$-adic setting, because unlike the usual absolute value and the nonarchimedean absolute value used in Theorem 1.1, with respect to divisibility, the $p$-adic modulus is a reverse ordering map, that is $|a|_{p} \geq|b|_{p}$ if $a$ divides $b$.

\section{EXAmples}

1) Let $K$ be a field, and let us consider now bivariate polynomials obtained by adding a linear factor in $Y$ to the polynomial $F(X, Y)$ in Corollary 1.2. We will first consider polynomials of the form

$$
F(X, Y)=f(X) \prod_{i=1}^{n}\left(Y-f_{i}(X)\right)+\sum_{i=1}^{n} g_{i}(X)\left(Y-f_{i}(X)\right),
$$

with $f, f_{1}, \ldots, f_{n}, g_{1}, \ldots, g_{n} \in K[X], f \neq 0$, and $\operatorname{deg} f_{i} \neq \operatorname{deg} f_{j}$ for $i \neq j$. It is easy to prove that if $F\left(X, f_{j}(X)\right) \neq 0$ for $j=1, \ldots, n$ and

$$
\max _{1 \leq i \leq n} \operatorname{deg} f_{i}+\max _{1 \leq i \leq n} \operatorname{deg} g_{i}<\frac{\lceil n / 2\rceil(\lceil n / 2\rceil-1)}{2},
$$

then $F$ must be irreducible over $K(X)$. If moreover $f_{1} \cdots f_{n} \neq 0$ then the same conclusion will hold if

$$
\max _{1 \leq i \leq n} \operatorname{deg} f_{i}+\max _{1 \leq i \leq n} \operatorname{deg} g_{i}<\frac{\lceil n / 2\rceil(\lceil n / 2\rceil+1)}{2}
$$


To prove this, we observe that $F\left(X, f_{j}(X)\right)=\sum_{i=1}^{n} g_{i}(X)\left(f_{j}(X)-f_{i}(X)\right)$, which shows that $\operatorname{deg} F\left(X, f_{j}(X)\right) \leq \max _{1 \leq i \leq n} \operatorname{deg} f_{i}+\max _{1 \leq i \leq n} \operatorname{deg} g_{i}$ for $j=1, \ldots, n$. The conclusion follows now by Theorem 1.1.

We note here that in Corollary 1.2 the degrees of $f_{1}, \ldots, f_{n}$ are only asked to be pairwise distinct, while in this case the degrees of $f_{1}, \ldots, f_{n}$ must be also bounded by the expression in the right side of (1) or (2).

2) One may also consider the case when the linear factor in $Y$ added to the polynomials in Corollary 1.2 does not depend explicitly on the polynomials $f_{1}, \ldots, f_{n}$. So let now

$$
F(X, Y)=f(X) \prod_{i=1}^{n}\left(Y-f_{i}(X)\right)+h(X) Y+g(X),
$$

with $f, g, h, f_{1}, \ldots, f_{n} \in K[X], f \neq 0$, and $\operatorname{deg} f_{i} \neq \operatorname{deg} f_{j}$ for $i \neq j$. Similarly, if one has $F\left(X, f_{j}(X)\right) \neq 0$ for $j=1, \ldots, n$ and

$$
\max \left\{\operatorname{deg} g, \operatorname{deg} h+\max _{1 \leq i \leq n} \operatorname{deg} f_{i}\right\}<\frac{\lceil n / 2\rceil(\lceil n / 2\rceil-1)}{2},
$$

then $F$ must be irreducible over $K(X)$. If moreover $f_{1} \cdots f_{n} \neq 0$ then the same conclusion will hold if

$$
\max \left\{\operatorname{deg} g, \operatorname{deg} h+\max _{1 \leq i \leq n} \operatorname{deg} f_{i}\right\}<\frac{\lceil n / 2\rceil(\lceil n / 2\rceil+1)}{2} .
$$

In this case $\operatorname{deg} F\left(X, f_{j}(X)\right) \leq \max \left\{\operatorname{deg} g, \operatorname{deg} h+\max _{1 \leq i \leq n} \operatorname{deg} f_{i}\right\}$ for $j=1, \ldots, n$, and the conclusion follows again by Theorem 1.1. Note that we obviously have $F\left(X, f_{j}(X)\right) \neq 0$ for $j=1, \ldots, n$ if, for instance, $g \neq 0$ and $g$ is not divisible by $h$.

3) Let us consider now the case when the degrees of $f_{1}, \ldots, f_{n}$ are not necessarily pairwise distinct. So let again

$$
F(X, Y)=f(X) \prod_{i=1}^{n}\left(Y-f_{i}(X)\right)+\sum_{i=1}^{n} g_{i}(X)\left(Y-f_{i}(X)\right),
$$

with $f, f_{1}, \ldots, f_{n}, g_{1}, \ldots, g_{n} \in K[X], f \neq 0$, and assume that $\operatorname{deg} f_{1} \leq \operatorname{deg} f_{2} \leq \cdots \leq \operatorname{deg} f_{n}$, and that the leading coefficients of $f_{1}, \ldots, f_{n}$ are pairwise distinct. If $F\left(X, f_{j}(X)\right) \neq 0$ for $j=1, \ldots, n$ and

$$
\operatorname{deg} f_{n}+\max _{1 \leq i \leq n} \operatorname{deg} g_{i}<\operatorname{deg} f_{2}+\operatorname{deg} f_{3}+\cdots+\operatorname{deg} f_{\lceil n / 2\rceil+1},
$$

then $F$ must be irreducible over $K(X)$.

Indeed, since $F\left(X, f_{j}(X)\right)=\sum_{i=1}^{n} g_{i}(X)\left(f_{j}(X)-f_{i}(X)\right)$, we deduce that $\operatorname{deg} F\left(X, f_{j}(X)\right) \leq$ $\operatorname{deg} f_{n}+\max _{1 \leq i \leq n} \operatorname{deg} g_{i}$ for $j=1, \ldots, n$, and the conclusion follows by Theorem 1.6.

4) Let now

$$
F(X, Y)=f(X) \prod_{i=1}^{n}\left(Y-f_{i}(X)\right)+h(X) Y+g(X)
$$


with $f, g, h, f_{1}, \ldots, f_{n} \in K[X], f \neq 0, \operatorname{deg} f_{1} \leq \operatorname{deg} f_{2} \leq \cdots \leq \operatorname{deg} f_{n}$, and assume that the leading coefficients of $f_{1}, \ldots, f_{n}$ are pairwise distinct. If $F\left(X, f_{j}(X)\right) \neq 0$ for $j=1, \ldots, n$ and

$$
\max \left\{\operatorname{deg} h+\operatorname{deg} f_{n}, \operatorname{deg} g\right\}<\operatorname{deg} f_{2}+\operatorname{deg} f_{3}+\cdots+\operatorname{deg} f_{\lceil n / 2\rceil+1},
$$

then $F$ must be irreducible over $K(X)$.

Here $\operatorname{deg} F\left(X, f_{j}(X)\right) \leq \max \left\{\operatorname{deg} h+\operatorname{deg} f_{n}, \operatorname{deg} g\right\}$ for $j=1, \ldots, n$, and the conclusion follows again by Theorem 1.6.

5) One may easily formulate similar irreducibility conditions for polynomials obtained by adding terms of higher degree with respect to $Y$ to the polynomials considered in Corollary 1.2 and Corollary 1.7. Consider, for instance, the polynomial

$$
F(X, Y)=f(X) \prod_{i=1}^{n}\left(Y-f_{i}(X)\right)+\sum_{i=0}^{k} h_{i}(X) Y^{i},
$$

with $f, f_{1}, \ldots, f_{n}, h_{0}, \ldots, h_{k} \in K[X], f \neq 0, \operatorname{deg} f_{1} \leq \operatorname{deg} f_{2} \leq \cdots \leq \operatorname{deg} f_{n}$, and assume that the leading coefficients of $f_{1}, \ldots, f_{n}$ are pairwise distinct. If $F\left(X, f_{j}(X)\right) \neq 0$ for $j=1, \ldots, n$ and

$$
\max _{0 \leq i \leq k}\left\{\operatorname{deg} h_{i}+i \operatorname{deg} f_{n}\right\}<\operatorname{deg} f_{2}+\operatorname{deg} f_{3}+\cdots+\operatorname{deg} f_{\lceil n / 2\rceil+1},
$$

then $F$ must be irreducible over $K(X)$.

Here $\operatorname{deg} F\left(X, f_{j}(X)\right) \leq \max _{0 \leq i \leq k}\left\{\operatorname{deg} h_{i}+i \operatorname{deg} f_{j}\right\}$ for $j=1, \ldots, n$, and the conclusion follows again by Theorem 1.6. Note that since $\operatorname{deg} f_{1} \leq \operatorname{deg} f_{2} \leq \cdots \leq \operatorname{deg} f_{n}$, (19) can not hold if $k \geq\lceil n / 2\rceil$, so one may obtain irreducibility criteria for polynomials of this type only if $k$ is sufficiently small. We also note that we obviously have $F\left(X, f_{j}(X)\right) \neq 0$ for $j=1, \ldots, n$, if for instance, the polynomial $\sum_{i=0}^{k} h_{i}(X) Y^{i}$ has no linear factors over $K(X)$.

Acknowledgements. This work was supported by CNCSIS-UEFISCDI, project PNIIIDEI 443, code 1190/2008, and by a project LEA CNRS Franco-Roumain Math-Mode.

\section{REFERENCES}

[1] M. Ayad, Irreducibility of $f(u(x), v(y))$, J. Algebra 279 (2004), 302-307.

[2] Y.F. Bilu, Quadratic factors of $f(x)-g(y)$, Acta Arith. 90 (1999) no. 4, 341-355.

[3] Y.F. Bilu, R.F. Tichy, The Diophantine equation $f(x)=g(y)$, Acta Arith. 95 (2000) no. 3, 261-288.

[4] A.I. Bonciocat, N.C. Bonciocat, Some classes of irreducible polynomials, Acta Arith. 123 (2006) no. 4, 349-360.

[5] A.I. Bonciocat, N.C. Bonciocat, A Capelli type theorem for multiplicative convolutions of polynomials, Math. Nachr. 281 (2008) no. 9, 1240-1253.

[6] A.I. Bonciocat, A. Zaharescu, Irreducibility results for compositions of polynomials in several variables, Proc. Indian Acad. Sci. (Math. Sci.) 115 (2005) no. 2, 117-126.

[7] N.C. Bonciocat, On an irreducibility criterion of Perron for multivariate polynomials, Bull. Math. Soc. Sci.Math. Roumanie 53 (101) (2010), no. 3, 213-217. 
[8] N.C. Bonciocat, A. Zaharescu, Irreducible multivariate polynomials obtained from polynomials in fewer variables, J. Pure Appl. Algebra 212 (2008), 2338-2343.

[9] M. Cavachi, M. Vâjâitu, A. Zaharescu, An irreducibility criterion for polynomials in several variables, Acta Math. Univ. Ostrav. 12 (2004), no. 1, 13-18.

[10] H.L. Dorwart, O. Ore, Criteria for the irreducibility of polynomials, Ann. of Math. 34 (1933), 81-99.

[11] H.L. Dorwart, O. Ore, Criteria for the irreducibility of polynomials, Ann. of Math. 35 (1934), 195.

[12] K. Győry, L. Hajdu, R. Tijdeman, Irreducibility criteria of Schur-type and Pólya-type, Monatsh. Math. DOI 10.1007/s00605-010-0241-9.

[13] S. Lang, Algebra, Graduate texts in mathematics 211, Springer, Berlin, New York, 2002.

[14] S. MacLane, A construction for absolute values in polynomial rings, Trans. Amer. Math. Soc. 40 (1936), 363-395.

[15] S. MacLane, The Schönemann - Eisenstein irreducibility criteria in terms of prime ideals, Trans. Amer. Math. Soc. 43 (1938), 226-239.

[16] L. Panaitopol, D. Ştefănescu, On the generalized difference polynomials, Pacific J. Math. 143 (1990) (2), 341-347.

[17] O. Perron, Neue Kriterien für die Irreduzibilität algebraischer Gleichungen, J. reine angew. Math. 132 (1907), 288-307.

[18] G. Pólya, Verschiedene Bemerkungen zur Zahlentheorie, Jahresber. Deutschen Math. Ver., 28 (1919), $31-40$.

[19] L.A. Rubel, A. Schinzel, H. Tverberg, On difference polynomials and hereditarily irreducible polynomials, J. Number Theory 12 (1980), 230-235.

[20] A. Schinzel, Polynomials with special regard to reducibility, Encyclopedia Math. Appl. 77, Cambridge Univ. Press, 2000.

[21] I. Schur, Problem 226, Archiv. der Math. und Physik 13 (1908) (3), p. 367.

[22] C. Shanok, Convex polyhedra and criteria for irreducibility, Duke Math. J., 2 (1936), 103-111.

Institute of Mathematics of the Romanian Academy, P.O. Box 1-764, Bucharest 014700, ROMANIA

E-mail address: Nicolae.Bonciocat@imar.ro, Mihai.Cipu@imar.ro

Université de Strasbourg, Mathématiques, 7, rue René Descartes, 67084 Strasbourg Cedex, France

E-mail address: yann.bugeaud@math.unistra.fr, maurice.mignotte@math.unistra.fr 